\title{
Psychological and Physical Effects of 10 Weeks Urban Forest Therapy Program on Dementia Prevention in Low-Income Elderly Living Alone
}

\author{
Hyun Jin Lee ${ }^{1}$ and Sung Ae Son ${ }^{2 *}$ \\ ${ }^{1}$ Interdisciplinary Program in Landscape Architecture, Seoul National University, Seoul 08826, Korea \\ ${ }^{2}$ Department of Forest Therapy, Graduate School of Chungbuk National University, Cheongju 28644, Korea
}

\begin{abstract}
Along with the aging society, the prevalence of dementia is also increasing. Dementia causes short-term memory loss as well as difficulties of performing daily activities and gradually causes suffering of the patients and their family. In spite of various programs for prevention of dementia of older people are being implemented, there is a lack of developing natural-based program for physical and mental health promotion. Therefore, it is necessary to develop programs for the elderly living alone who are more vulnerable to dementia because of their social and economic isolation. The purpose of this study was to develop a natural-based program and investigate the effects of 10 weeks forest therapy program for dementia prevention to improve the psychological and physical health of the elderly living alone. The experimental subjects were 30 elderly (aged 65 or older) and 31 elderly participated in control group. The Stress response, depressive symptoms, weight, body mass index (BMI), fat mass and muscle mass were measured for pre and post test. The results showed that the experimental group showed subjective stress relief ( $\mathrm{t}=5.249, p=.000)$, improvement in symptoms of depression $(\mathrm{t}=4.152, p=.000)$, and decreases in weight $(\mathrm{t}=2.686, p=.012), \mathrm{BMI}(\mathrm{t}=2.629, p=.014)$ and fat mass $(\mathrm{t}=2.918, p=.007)$ after the forest therapy program. The experimental group showed lower stress reactions $(\mathrm{t}=-7.185, p=.000)$ and less depressive symptoms $(\mathrm{t}=-5.303, p=.000)$ than control group after participating the program. These results suggest that periodic forest exposure can help having less stressful and depressive status than non-forest exposure and the forest therapy program can reduce participants' psychological and physical risk factors of dementia.
\end{abstract}

Keywords: forest healing, health benefits, nature-based intervention, underprivileged older people

\section{Introduction}

South Korea entered the aging society in 2017 with the elderly population reaching $14.0 \%$ of the entire population (MOIS, 2017). Along with the increase of the elderly population, the number of elderly living alone is also constantly increasing. There are as many as 1.44 million elders living alone as of 2016, which will rapidly increase to 3.43 million by 2035 (KOSIS, 2016).

The forest therapy program in this study was run by Healing Forest Cooperative with the support of Korea Forest Service, Korea Forest Welfare Institute, and Lottery Fund (Green Fund).

Received: October 30, 2018, Revised: November 5, 2018, Accepted: November 19, 2018

First author: Hyun Jin Lee, E-mail: hlhl411@gmail.com, ORCID: 0000-0001-5061-2716

*Corresponding author: Sung Ae Son, E-mail: adept13@naver.com, ORCID: 0000-0003-0993-2042 
Due to the aging society, the dementia populations are also skyrocketing globally, which leads to increase in social costs (Wimo et al., 2017). Once occurred, dementia is accompanied by great burden and pain with long-term degeneration, and the low-income elderly living alone may be more vulnerable to onset of dementia in case they lack individual and social support as well as network (Ennis et al., 2014). However, since the cure for dementia is yet to be developed, it is best to reduce the risks or delay the occurrence with a healthy lifestyle (Barnard et al., 2014). Therefore, it is necessary to perceive dementia not as an individual problem but as a social problem, thereby increasing preventive intervention and developing and expanding programs to prevent and manage dementia for high risk groups.

Forest therapy is one of the nature-based therapies that promote physical and mental health using various elements of forest environment such as landscape, sound, phytoncide, anion and sunlight (Yu et al., 2017). Many studies on the effects of forests on physical and mental health proved that forests reduced anxiety and depression, lifted the mood (Ochiai et al., 2015; Yu et al., 2017), and improved cognitive functions (Bratman et al., 2015), while also showing physiological effects on chronic diseases by lowering blood pressure for hypertensive patients (Mao et al., 2012; Song et al., 2017) and blood sugar level for diabetics (Song et al., 2017). Furthermore, as depression, cognitive functions, stress and chronic diseases are known to be the risks of dementia (Deckers et al., 2015), intervention of forest therapy programs may be effective for prevention of dementia and clearly have positive impacts on reducing social costs. However, despite the effects of forest therapy programs on reducing risks of dementia, there is insufficient research on nature-based programs targeting the low-income elderly living alone that are particularly vulnerable to dementia. Meanwhile, urban forests have diverse features among regions but they provide a variety of vegetations and spaces to be used as physical and mental healing places for urban dwellers (Han and Koo, 2018). Therefore, it is necessary to develop forest therapy programs using accessible urban forests for the elderly living alone that are physically and economically vulnerable to long distance travel.

In this context, this study is to develop an urban forest therapy program to prevent dementia for the low-income elderly living alone, and verify the effectiveness of the program by determining its psychological effects on stress and depressive symptoms and physical effects on body mass index (BMI), fat mass, and muscle mass.

\section{Research Methods}

\section{Research design}

This study is an experiment with nonequivalent control group pretest-posttest design to verify the health promotion effect of subjects by providing a forest therapy program to prevent dementia for low-income elderly living alone in the city.

\section{Subjects}

The subjects of this study are the elderly aged 65 and above living alone in $\mathrm{N}$-gu, Seoul that are beneficiaries of medical care and are capable of physical activities, who participated in the 'Urban Forest Therapy Program for Dementia Prevention' carried out at an urban forest. They were recruited at the community service centers of A-dong and B-dong that are unrelated to this program, and they expressed their voluntary will to participate after listening to sufficient explanation about the research objectives and methods. The subjects were limited to those capable of physical activities in order to participate in the program, and those with sensory disturbance, cognitive disorder and mental disorder were excluded as they may create confusion in the results. The samples of this study were limited to 02 or less from each dong considering the number that can be controlled by two forest therapists, and thus ultimately 30 subjects participated in the 
experimental group (15 from A-dong, 15 from B-dong) and 31 in the control group (15 from A-dong, 16 from B-dong). Their mean age was $76.3 \pm 4.3$ in the experimental group, and 77.0 \pm 8.0 in the control group.

Before collecting data, the researchers identified themselves, described the research objectives and procedures, and explained to the subjects that the collected data will be used only for research purposes with their confidentiality guaranteed. They also explained that the data will be discarded once the research is concluded, and that the subjects have the right to refuse participation in the middle of the study, and had the subjects sign the consent form.

\section{Measurement tools and methods}

This study measured the effects of the forest therapy program in the psychological and physical aspects. First, it is to increase the reliability of results with complex analysis of subjective and objective indexes, because psychological factors such as depression and stress and physical factors such as chronic diseases serve as risk factors of dementia. Second, it is to compare the dementia prevention effects on participants exposed to the forest environment with non-participants by evaluating the objective physical influence in addition to subjective psychological differences among participants in measuring the participation effects of the long-term regular forest therapy program.

A pretest-posttest evaluation of the survey was conducted on the experimental group in Session 1 and Session 10 of the program, and the InBody test was performend on April 7 (pretest) and July 24-25 (posttest). The control group took the pretest survey and InBody test on April 10-11, and then received posttest on July 24-25.

\section{Psychological measurement index}

First, Stress Response Inventory (SRI) developed by Koh et al. (2000) was used to measure the emotional, physical, cognitive and behavioral responses toward stress, which were rated on a 5-point Likert scale. The reliability coefficient (Cronbach- $\alpha$ ) of the scale was .648, showing satisfactory internal consistency of .6 or higher. The total score of 50 points or below is regarded as 'low stress,' 51-80 as 'need self-reflection and mind control,' 81-120 as 'high stress and need control,' and 120 or above as 'severe stress and need counseling.' Second, to evaluate loneliness and depression in daily life, Korean Beck Depression Inventory-II (K-BDI-II) by Rhee et al. (1995) is used. The reliability coefficient (Cronbach- $\alpha$ ) of the scale was .772, consisting of total 21 items on sorrow, criticism, worthlessness, sleep, and appetite. The total score of 9 points is regarded as 'not depressed,' 10-15 as 'mildly depressed,' 16-23 as 'depressed,' and 24 or above as 'severely depressed and need consultation of specialist.' Psychological data were collected in the form of a self-report questionnaire from the experimental group on the day of the beginning and end of the program, and from the control group at the $\mathrm{N}$-gu branch office of the community health center.

\section{Physical measurement index}

For pretest-posttest physical measurement, a body composition analyzer (InBody ACCUNIQ) was used to measure the changes in body mass index (BMI), fat mass and muscle mass. First, BMI is an index that evaluates obesity using height and weight, and higher BMI indicates higher possibility of getting a disease. It is measured by dividing weight (kg) by height squared (m), with 18.5 regarded as underweight, 18.5-22.9 as normal weight 23-24.9 as overweight, 25-29.9 as obese, and 30 or above as extremely obese. Second, fat mass is the amount of body fat in weight, and obesity is determined by how much excess fat is in the body, not by the weight. Third, muscle mass is measured by the index proportionate to basal metabolism and energy expenditure. Physical data were collected from both the experimental and control group at the N-gu branch office of the community health center for pretest-posttest evaluation. 


\section{Forest therapy program}

The urban forest therapy program to prevent dementia was carried out from April 18 to July 12, 2018 from 10 a.m. to 1 p.m. every week in total 9 sessions at the urban forest located in N-gu, Seoul targeting A-dong and B-dong. In the last Session 9, subjects from both A-dong and B-dong spent the night at $\mathrm{S}$ Therapy Forest in Gyeonggi-do. The main activities of this program were 'warming up and forest walking,' 'five senses meditation' and 'traditional play.' Warming up and forest walking are effective for managing risk factors of depression and dementia through physical stimulation and brain stimulation activities (Lee, 2009; Kim and Ahn, 2015). Forest meditation reduces unnecessary attention and makes people concentrate on their bodies, and is effective for stimulating the five senses such as vision, hearing and smell (Lee and Shin, 2015). In particular, the five senses deteriorate after midlife, which also leads to decline of cognitive functions, which is why meditation using the five senses helps induce cognitive stimulation of the elderly and relax their emotions (Yoo, 2010). Traditional play is effective for relaxing emotions and maintaining or promoting the remaining physical abilities of the elderly by recalling the emotions and memories of childhood through traditional play using natural objects (Seo, 2007). Based on the results of previous studies, this study organized the program as shown in Table 1, and the details are provided in Table 2. Each session consists of introduction (20-30 minutes, walking, stretching, five senses meditation, etc.), development (120 minutes, traditional play, lunch), and wrap-up (20-30 minutes, breathing, tasks, etc.).

\section{Analysis method}

SPSS WIN 18.0 was used for statistical analysis of the collected data, and the independent t-test is used to test the homogeneity of the two groups. For hypothesis testing, the paired t-test is used to verify pretest-posttest differences of the program after checking normal distribution of each dependent variable, and the independent t-test was used to compare the results between the experimental group and control group.

\section{Results and Discussion}

\section{Homogeneity test of variables}

There was no significant difference between the two groups considering their psychological factors. However, in physical factors, the control group had higher weight, body mass index and fat mass than the experimental group (weight: $\mathrm{t}=-2.393, p=.02$, body mass index: $\mathrm{t}=-3.062, p=.003$, fat mass: $\mathrm{t}=-2.504, p=.015$ ) (Table 3 ).

Table 1. Forest therapy program for preventing dementia

\begin{tabular}{cr}
\hline Session & Program contents \\
\hline 1 & Warming up, forest walking, herbal therapy, assignment \\
2 & Warming up, forest walking, five senses meditation, communicating with nature \\
3 & Warming up, forest walking, five senses meditation, traditional play, assignment \\
4 & Warming up, forest walking, five senses meditation, traditional play \\
5 & Warming up, forest walking, five senses meditation, making traditional snack, assignment \\
6 & Warming up, forest walking, five senses meditation, traditional play \\
7 & Warming up, forest walking, five senses meditation, traditional play, assignment \\
8 & Warming up, forest walking, five senses meditation, making traditional snack \\
9 & Warming up, forest walking, five senses meditation, aromatic hand massage, feedback \\
10 & Forest walking, water therapy, planning healthy life, writing a letter to me, five senses meditation, \\
\hline
\end{tabular}


Table 2. Activity details of forest therapy program for preventing dementia

\begin{tabular}{|c|c|}
\hline Contents & Activities \\
\hline Warming up & Body tapping, eyeball turning, hand clapping, etc. \\
\hline Forest walking & Walking slowly while listening to the sound of the forest, looking at the trees and feeling the wind \\
\hline Communicating with nature & Tree hugging, observing the shape of trees \\
\hline Herbal therapy & Making wooden necklace with herb leaf, drinking herbal tea \\
\hline Five senses meditation & Focusing on listening to, looking at and feeling the nature, deep breathing \\
\hline Traditional play & Stone hitting game, stacking up wood stick game, etc. \\
\hline Making traditional snack & Making canape with flowers and fruits, making natural jelly \\
\hline Aromatic hand massage & Giving hand massage with aromatic oil each other \\
\hline Assignment & Making a pet tree \& hugging, checking health care daily log, etc. \\
\hline Feedback \& Question & Talking about experiences and asking questions \\
\hline Water therapy & Exercising and walking in the water tube \\
\hline $\begin{array}{l}\text { Planning health life, } \\
\text { writing a letter to me, } \\
\text { communicating with others }\end{array}$ & Talking and sharing about health promotion \\
\hline
\end{tabular}

Table 3. Baseline of experimental $(n=30)$ and control group $(n=31)$ in psychological and physical indicators

\begin{tabular}{|c|c|c|c|c|c|}
\hline Indicator & Variable & Group & $\mathrm{M}(\mathrm{SD})$ & $\mathrm{t}$ & $p$ \\
\hline \multirow[t]{4}{*}{ Psychological } & $\mathrm{SRI}^{\mathrm{z}}$ & Experimental & $82.57(28.36)$ & -0.694 & .490 \\
\hline & & Control & $87.58(28.03)$ & & \\
\hline & K-BDI-II & Experimental & 17.37(10.95) & -1.153 & .254 \\
\hline & & Control & 20.74(11.90) & & \\
\hline \multirow[t]{8}{*}{ Physical } & Weight(kg) & Experimental & $58.12(8.42)$ & -2.393 & $.020^{*}$ \\
\hline & & Control & $64.40(11.84)$ & & \\
\hline & Body mass index $\left(\mathrm{kg} / \mathrm{m}^{2}\right)$ & Experimental & $23.97(2.37)$ & -3.062 & $.003^{* *}$ \\
\hline & & Control & $26.70(4.28)$ & & \\
\hline & Fat mass(kg) & Experimental & $17.93(3.99)$ & -2.504 & $.015^{*}$ \\
\hline & & Control & $21.65(7.11)$ & & \\
\hline & Muscle mass(kg) & Experimental & $37.22(6.93)$ & -1.068 & .290 \\
\hline & & Control & $39.14(7.11)$ & & \\
\hline
\end{tabular}

${ }^{\mathrm{z}} \mathrm{SRI}=$ Stress Response Inventory; K-BDI-II= Korean Beck Depression Inventory-II

${ }^{*} p<.05,{ }^{* *} p<.01$

\section{Pretest-posttest comparison of the experimental group}

Comparing the pretest and posttest results of the experimental group, there was a significant difference with a remarkable decrease in stress reaction and depressive symptoms of psychological factors (SRI: $\mathrm{t}=5.249, p=.000$, K-BDI-II: $\mathrm{t}=4.152, p=.000$ ). This considers the possibility of the program and place. First, the fact that the main activity of this program consists of traditional play that is in the form of team-based games for the subjects to increase social ties and have fun increased social support and relieved loneliness of the elderly living alone, and their memory, recollection and cognitive functions improved as they re-experienced the games that are familiar from their childhood. Moreover, regularly meditating with the five senses awakened the deteriorated five senses and reduced stress and depressive symptoms with 
emotional and mental relaxation. This result is consistent with that of previous studies proving the effects of the forest therapy program on emotional stability and general stress relief (Ochiai et al., 2015; Yu et al., 2017). In terms of place, the urban forest within the living circle that is close to residential area provides familiar sense of place and improves the quality of life for urban dwellers (Han and Koo, 2018), thereby emotionally and mentally stabilizing the participants without resistance or incompatibility. It is also highly accessible for the elderly and thus effective in making them constantly participate in the long-term program.

For physical factors, there was a significant decrease in all of weight $(\mathrm{t}=2.686, p=.012)$, body mass index $(\mathrm{t}=2.629$, $p=.014)$, and fat mass $(\mathrm{t}=2.918, p=.007)$. However, muscle mass did not show a statistically significant difference despite the slight increase (Table 4). This may be related to the fact that the program for the elderly consists of mostly lowintensity physical activities such as stretching, walking and fine motor skills activities (Asp et al., 2017).

\section{Pretest-posttest comparison of the control group}

Unlike the experimental group, the control group showed a significant increase in stress reaction of psychological factors (SRI: $\mathrm{t}=-3.787, p=.001)$, and a significant decrease in fat mass of physical factors $(\mathrm{t}=2.440, p=.021)$. On the other hand, there was a slight increase in depressive symptoms (K-BDI-II: $\mathrm{t}=-0.713, p=.481)$ and muscle mass $(\mathrm{t}=-1.627$, $p=.114$ ), but there was no statistically significant difference (Table 5).

\section{Posttest comparison of the experimental and control group}

The physical variables of both groups were not homogeneous, and thus as a result of comparing stress reaction and

Table 4. Comparison of pre- and posttest result in psychological and physical indicators of experimental group ( $\mathrm{n}=30$ )

\begin{tabular}{|c|c|c|c|c|c|}
\hline Indicator & Variable & Pre-M(SD) & Post-M(SD) & $\mathrm{t}$ & $p$ \\
\hline \multirow[t]{2}{*}{ Psychological } & $\mathrm{SRI}^{\mathrm{z}}$ & $82.57(28.36)$ & $58.67(15.98)$ & 5.249 & $.000^{\text {**** }}$ \\
\hline & K-BDI-II & $17.37(10.95)$ & $10.50(5.51)$ & 4.152 & $.000^{* * *}$ \\
\hline \multirow[t]{4}{*}{ Physical } & Weight(kg) & $58.12(8.42)$ & $57.54(8.01)$ & 2.686 & $.012^{*}$ \\
\hline & Body mass index $\left(\mathrm{kg} / \mathrm{m}^{2}\right)$ & $23.97(2.37)$ & $23.75(2.32)$ & 2.629 & $.014^{*}$ \\
\hline & Fat mass(kg) & $17.93(3.99)$ & $17.32(4.09)$ & 2.918 & $.007^{* *}$ \\
\hline & Muscle mass(kg) & $37.22(6.93)$ & $37.30(6.71)$ & -0.454 & .653 \\
\hline
\end{tabular}

${ }^{\mathrm{z}} \mathrm{SRI}=$ Stress Response Inventory; K-BDI-II= Korean Beck Depression Inventory-II

${ }^{*} p<.05,{ }^{* *} p<.01$

Table 5. Comparison of pre- and posttest result in psychological and physical indicators of control group ( $\mathrm{n}=31$ )

\begin{tabular}{llccccc}
\hline Indicator & \multicolumn{1}{c}{ Variable } & Pre-M(SD) & Post-M(SD) & $\mathrm{t}$ & \multicolumn{1}{c}{$p$} \\
\hline Psychological & SRI $^{\mathrm{z}}$ & $87.58(28.03)$ & $103.61(30.43)$ & -3.787 & $.001^{* *}$ \\
& K-BDI-II & $20.74(11.90)$ & $22.45(11.08)$ & -0.713 & .481 \\
Physical & Weight(kg) & $64.40(11.84)$ & $63.41(11.13)$ & 1.764 & .088 \\
& Body mass index $\left(\mathrm{kg} / \mathrm{m}^{2}\right)$ & $26.70(4.28)$ & $26.25(3.93)$ & 1.735 & .093 \\
& Fat mass $(\mathrm{kg})$ & $21.65(7.11)$ & $20.57(6.46)$ & 2.440 & $.021^{*}$ \\
& Muscle mass(kg) & $39.14(7.11)$ & $39.60(7.23)$ & -1.627 & .114 \\
\hline
\end{tabular}

${ }^{\mathrm{z}} \mathrm{SRI}=$ Stress Response Inventory; K-BDI-II= Korean Beck Depression Inventory-II

${ }^{*} p<.05,{ }^{* *} p<.01$ 
depressive symptoms of psychological factors, the experimental group showed a significantly lower value than the control group, thereby improving stress reaction and depressive symptoms (SRI: $\mathrm{t}=-7.185, p=.000, \mathrm{~K}-\mathrm{BDI}-\mathrm{II}: \mathrm{t}=-5.303, p=.000$ ). Meanwhile, there are various possibilities for the fact that the experimental group showed a decrease and the control group showed an increase in contrary. First, there is a difference in place between the experimental group (forest) and control group (health center) in taking the psychological index survey. Second, the experimental group showed an increase in intimacy within the group through regular forest experience, thereby affecting subjective psychological stability in the posttest measurement.

\section{Conclusion}

This study compared the effects of the urban forest therapy program for the elderly living alone on dementia prevention by measuring and comparing the differences of psychological factors and physical factors. First, the experimental group that experienced 10 weeks of regular therapy program showed a significant decrease in stress reaction and depressive symptoms of psychological factors, whereas the control group showed an increase. Second, physical factors of the two groups are not homogeneous, but the experimental group showed a significant decrease in weight, BMI and fat mass of physical factors after the program. This result implies that for the low-income elderly living alone, the nature-based therapy program is effective in not only psychologically preventing dementia by reducing stress and depressive symptoms but also physically preventing dementia by reducing weight and fat mass.

However, this study has a few limitations. First, considering the subjective survey measurement, the environmental characteristics where the survey is conducted and intimacy of the participants may affect the study results of the experimental group and control group, which is why it is necessary to consider providing the same place or psychological distance. Second, characteristics of the samples must also be considered. The participants of this study were elders living alone in the city and receiving medical care. However, considering the residential characteristics of the city or psychological and nutrition status of individuals, the 61 participants of this study cannot fully represent all elders living alone. Therefore, it is necessary to be cautious about applying the results of this study to similar or different groups. Moreover, these results did not consider variables other than the frequency of using the urban forest, which is why it is necessary to consider the fact that the results may be affected by other mediating variables such as the present health condition of the subjects.

Despite the limitations, the results of this study have significance as it empirically supported the hypothesis that the regular urban forest therapy program consisting of 'warming up and forest walking', 'five senses meditation'and 'traditional play' as the main activities for dementia prevention reduces stress, depressive symptoms, weight and fat mass of the elderly living alone through physical activities. However, considering that psychological interpretation of a given situation performs a crucial role in promoting social behavior, more research is needed in order to determine the mechanisms of psychological and physical effects of the program. Therefore, psychological interpretation such as reduction of stress and depressive symptoms through the program must be examined more in depth by using qualitative research methods such as participant observation and interview as well as physiological and psychological tests. Furthermore, future research must expand the scope of participants to support use of forest therapy programs and urban forests as resources of green welfare, thereby verifying the validity of this study.

\section{References}

Asp, M., B. Simonsson, P. Larm, and A. Molarius. 2017. Physical mobility, physical activity, and obesity among elderly: 
findings from a large population-based Swedish survey. Public health 147:84-91. DOI:10.1016/j.puhe.2017.01.032

Barnard, N.D., A.I. Bush, A. Ceccarelli, J. Cooper, C.A. de Jager, K.I. Erickson, G. Fraser, S. Kesler, S.M. Levin, B. Lucey, M.C. Morris, and R. Squitti. 2014. Dietary and lifestyle guidelines for the prevention of Alzheimer's disease. Neurobiol. Aging 35:S74-S78. DOI:10.1016/j.neurobiolaging.2014.03.033

Bratman, G.N., G.C. Daily, B.J. Levy, and J.J. Gross. 2015. The benefits of nature experience: Improved affect and cognition. Landsc. Urban Plan. 138:41-50. DOI:10.1016/j.landurbplan.2015.02.005

Deckers, K., M.P. van Boxtel, O.J. Schiepers, M. de Vugt, J.L. Muñoz Sánchez, K.J. Anstey, C. Brayne, J.F. Dartigues, K. Engedal, M. Kivipelto, K. Ritchie, J.M. Starr, K. Yaffe, K. Irving, F.R. Verhey, and S. Köhler. 2015. Target risk factors for dementia prevention: a systematic review and delphi consensus study on the evidence from observational studies. Int. J. Geriatr. Psychiatry 30(3):234-246. DOI:10.1002/gps.4245

Ennis, S.K., E.B. Larson, L. Grothaus, C.D. Helfrich, S. Balch, and E.A. Phelan. 2014. Association of living alone and hospitalization among community-dwelling elders with and without dementia. J. Gen. Intern. Med. 29(11):1451-1459. DOI:10.1007/s11606-014-2904-z

Han, I.D. and C.D. Koo. 2018. The effect of forest healing program on the resilience of elderly people in urban forest. J. People Plants Environ. 21(4):293-303. DOI:10.11628/ksppe.2018.21.4.293

Kim, S.H. and E.J. Ahn. 2015. A study on developing gymnastic program for seniors with mild dementia \& walking problems: Measuring its effects. J. Sport Leis. Stud. 59(1):601-618. Retrieved from http://www.kssls.co.kr

Koh, K.B., J.K. Park, and C.H. Kim. 2000. Development of the Stress Response Inventory. J. Korean Neuropsychiatr. Assoc. 39(4):707-719.

KOSIS(Korean Statistical information service). 2016. Future population estimation. Retrieved from www.kosis.kr

Lee, K.O. 2009. The effects of the gymnastic exercise and walking program on body composition, depression and risk factors of dementia in the elderly women. Korean J. Sport Sci. 18(2):1011-1026.

Lee, Y.J. and C.S. Shin. 2015. Effects of forest walking meditation on mood states and self-awareness in middle-aged women. J. Korean Inst. For. Recreat. 19(3):19-25.

Mao, G.X., Y.B. Cao, X.G. Lan, Z.H. He, Z.M. Chen, Y.Z. Wang, X.L. Hu, Y.D. Lv, G.F. Wang, and J. Yan. 2012. Therapeutic effect of forest bathing on human hypertension in the elderly. J. Cardiol. 60(6):495-502.

MOIS (Ministry of the Interior and Safety). 2017. 2017 Demographics. Retrieved from www.mois.go.kr

Ochiai, H., H. Ikei, C. Song, M. Kobayashi, T. Miura, T. Kagawa, Q. Li, S. Kumeda, M. Imai, and Y. Miyazaki. 2015. Physiological and psychological effects of a forest therapy program on middle-aged females. Int. J. Environ. Res. Public Health 12(12):15222-15232. DOI:10.3390/ijerph121214984

Rhee, M.K., Y.H. Lee, H.Y. Jung, J.H. Choi, S.H. Kim, Y.K. Kim, and S.K. Lee. 1995. A standardization study of Beck Depression Inventory II - Korean Version(K-BDI): Validity. Korean J. Psychopathol. 4(1):96-104.

Seo, J.O. 2007. An empirical study on reality effectiveness of improving cooperation activity of therapeutic recreation to the old dementia : Focusing on tradition game program. Master's thesis, Sangmyung University, Seoul, Korea.

Song, C., H. Ikei, and Y. Miyazaki. 2017. Sustained effects of a forest therapy program on the blood pressure of office workers. Urban For. Urban Green. 27:246-252.

Wimo, A., M. Guerchet, G.C. Ali, Y.T. Wu, A.M. Prina, B. Winblad, L. Jönsson, Z. Liu, and M. Prince. 2017. The worldwide costs of dementia 2015 and comparisons with 2010. Alzheimers Dement. 13(1):1-7. DOI:10.1016/j.jalz.2016.07.15.

Yoo, S.H. 2010. A study on the environmental evaluations and suggestions of nursing homes in the aspects of environmental psychological behaviors of elderly with dementia. Master's thesis, Chung-Ang University, Seoul, Korea.

Yu, C.P., C.M. Lin, M.J. Tsai, Y.C. Tsai, and C.Y. Chen. 2017. Effects of short forest bathing program on autonomic nervous system activity and mood states in middle-aged and elderly individuals. Int. J. Environ. Res. Public Health 14(8):897. DOI:10.3390/ijerph14080897 